\title{
Familial 5q12.3 Microdeletion: Evidence for a Locus Associated with Epilepsy
}

\author{
Chiara Gnan $^{\mathrm{a}}$ Alessandra Franzoni ${ }^{\mathrm{a}}$ Federica Baldan ${ }^{\mathrm{a}}$ Nadia Passon $^{\mathrm{a}}$ \\ Giuseppe Damante $^{\mathrm{a}, \mathrm{b}}$ Patrizia Dello Russo ${ }^{a}$

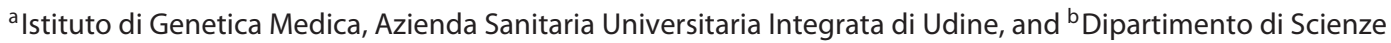 \\ Mediche e Biologiche, Università di Udine, Udine, Italy
}

\section{Key Words}

Array CGH · Epilepsy · Interstitial microdeletion 5q

\begin{abstract}
The clinical use of array comparative genomic hybridization (array CGH) has allowed the identification of very rare deletion and duplication disorders, such as 5q12 deletion syndrome (OMIM 615668) described as a contiguous gene deletion syndrome of chromosome $5 q 12$. Chromosome microdeletions including band $5 q 12$ have rarely been reported and have been associated with different phenotypes showing postnatal growth restriction, intellectual disability, epileptic seizures, hyperactivity, and ocular abnormalities. In this study, we describe a family in which array-CGH analysis revealed the presence of an interstitial microdeletion spanning approximately $2.9 \mathrm{Mb}$ in the $5 q 12$ region. The microdeletion is associated with epilepsy in the father and 2 siblings (a boy and a girl). So far, this is the first report in which a familial microdeletion 5q12 manifests in epilepsy. We suggest that this familial microdeletion could delineate a locus for susceptibility to epilepsy.

(c) 2017 S. Karger AG, Basel
\end{abstract}

(c) 2017 S. Karger AG, Basel

E-Mail karger@karger.com

www.karger.com/msy
In the last years, the strategy for the detection of submicroscopic chromosomal anomalies has been revolutionized by the introduction of array comparative genomic hybridization (array CGH). Whole genome array $\mathrm{CGH}$ has made it possible to define many cases of copy number variations in patients with unexplained intellectual disability (ID), behavioral problems, and other similar features. Among these rare genomic disorders, the interstitial deletion encompassing the $5 \mathrm{q} 12$ region is included, associated with different clinical phenotypes not always consistent. Until now, only 6 sporadic patients with microdeletion in $5 \mathrm{q} 12$ have been reported. They shared some clinical characteristics, such as postnatal growth retardation, ID, behavioral abnormalities (hyperactivity), nonspecific ocular defects, and facial dysmorphism [Holder and Cheung, 2015]. Only 3 patients showed epilepsy as an additional clinical finding [Jaillard et al., 2011; Cetin et al., 2013].

Holder and Cheung [2015] described a 9-year-old boy referred for examination because of speech delay, who had a 4.0-Mb de novo deletion in 5q12.3q13.2, while Jaillard et al. [2011] reported 4 unrelated children with developmental delay/cognitive disability and facial dysmorphism, who were found to have heterozygous de novo deletions in 5q12.1, spanning from 5.7 to $17.2 \mathrm{Mb}$. Only

Prof. Giuseppe Damante

Dipartimento di Scienze Mediche e Biologiche, Università di Udine Piazzale Kolbe 4

IT-33100 Udine (Italy)

E-Mail giuseppe.damante@uniud.it 
2 of the patients described by Jaillard et al. [2011] have reported seizures. Furthermore, Cetin et al. [2013] described a 2-year-old boy with ID and developmental delay in whom a 5q12.1q12.3 de novo microdeletion was detected. He presented with facial dysmorphism, psychomotor impairment, and incurred in epileptic seizures on a regular basis.

Here, we report the first case of a familial microdeletion in 5q12.3 associated with epilepsy. In this study, we analyzed a 13-year-old boy without dysmorphic features, with moderate ID, speech and motor delay, and epilepsy. He inherited the 5q12.3 microdeletion from his father (with epilepsy). Moreover, he has an older sister suffering from a moderate form of epilepsy, who showed the same microdeletion.

\section{Patient and Methods}

\section{Present Case}

The index patient, a 13-year-old boy, is the second-born child of nonconsanguineous Caucasian parents; he has a 16-year-old sister. He was born after a full-term uneventful pregnancy. The proband was born with birth parameters within normal range. At genetic examination, his weight, height, and head circumference were $64.5 \mathrm{~kg}$ (90-95th percentile), $158 \mathrm{~cm}$ (50-75th percentile), and $55.8 \mathrm{~cm}$ (75th percentile), respectively. There were no signs of dysmorphism. The proband was able to speak and walk at about 2-3 years of age, and around 7 years, he had the first episode of seizure. He was referred to genetic counseling for his mild ID and behavioral problems. Now he attends middle school, but he requires a support teacher. The father and the sister of our proband are epileptic and receiving antiepileptic drug treatment. No evident cognitive impairment was present in the father; however, he showed difficulties in relationships with the other family members. The sister has a very mild case of ID, also requiring a support teacher. The boy's mother had 2 prior miscarriages due to unknown reasons.

\section{Methods}

Array CGH

Blood samples from the patient, his parents, and sister were drawn after informed consent. Genomic DNA was isolated from peripheral blood using the QIAamp DNA Blood Midi Kit (Qiagen $\mathrm{GmbH}$, Hilden, Germany).

Array-CGH analysis was carried out following the manufacturer's instructions using Agilent Human Genome CGH oligonucleotide array 180k (Human Genome CGH Microarray Kit 180k; Agilent Technologies, Santa Clara, CA, USA). The microarray includes 180,000 oligonucleotide probes at the spacing average of $13,000 \mathrm{bp}$. Genomic DNA samples and reference samples (Coriell Institute, Camden, NJ, USA) were labeled Cy3 and Cy5, respectively, using Agilent Enzymatic Labeling protocol. After the hybridization protocol, slides were scanned using Agilent G2505 Scanner. Image files were analyzed by Agilent Feature Extraction, Genomic Workbench 6.5.018 Lite Edition Software, and genomic coordinates were evaluated according to GRCh37/hg19. Genes located in the deleted area were investigated using the UCSC genome browser database (http://genome.ucsc.edu, hg19).

Quantitative Real-Time PCR

Quantitative PCR was performed using 7300 Real-Time PCR System (Applied BioSystems, Foster City, CA, USA) on the samples of our proband and his family to confirm and to determine the inheritance of the chromosomal aberration. We used primers directed to one of the genes present in the deleted region (SREK1IP1), selected for the purpose of confirming the deletion. The sequences of the primers are: F: $5^{\prime}$-AGGCCGAGTCAGCTCTAGTGAT-3; R: 5'-CAAGTAATGAAATGAGCAGTGATAACAG-3' . As reference, we used the $A L B$ gene, amplified with the following primers: F: 5'-ATGCTGCACAGAATCCTTGGT-3'; R: 5'-TCATCGACTTCCAGAGCTGAAA- $3^{\prime}$.

\section{Results}

The array-CGH analysis identified the presence of a 2.8-Mb-deleted 5q12.3 region (spanning from 63,582,183 to $66,449,431 \mathrm{bp}$; hg 19; Fig. 1A), which included 19 RefSeq genes, of which 8 are comprised in the OMIM database (RNF180, RGS7BP, ADAMTS6, CENPK, TRIM23, $N L N, E R B B 2 I P$, and SREK1). None of these deletions are thought to be individually pathogenic; for this reason, we consider that the epileptic seizures are due to contiguous gene deletions.

In order to confirm the presence of the deletion by a different assay, quantitative PCR was performed. This procedure validated the presence of the deletion in the proband. Further analysis of his parents and sister revealed a paternal origin of the deletion and showed the same deletion in the sister (Fig. 1B).

\section{Discussion}

Here, we describe a family with a $2.8-\mathrm{Mb}$ deletion in the $5 \mathrm{q} 12.3$ region using array-CGH analysis. In this family, the presence of the deletion is associated with epilepsy. We first found the deletion in our patient presenting with epilepsy and mild ID. Quantitative PCR confirmed the deletion in the boy's father and sister. Both have manifested epileptic seizures, and up to now, are subjected to drug therapy. Our patient's most prominent features are poor language and developmental delay; he was not able to speak and walk independently until he was 3 years old.

The OMIM database (www.omim.org) reports a chromosome 5q deletion syndrome (OMIM 615668) described as a contiguous gene deletion syndrome. The 
Fig. 1. Array CGH and quantitative PCR. A Array-CGH profile using Genomic Workbench 6.5.018 Lite Edition Software. The value of zero represents equal fluorescence intensity ratio between the sample and reference gDNA. Copy number losses shift the ratio to the left and copy number gains to the right. Red and green dots indicate values above or below the 0 baseline, respectively. B Values of quantitative PCR in the deleted region. Each bar indicates the mean values $\pm 3 \mathrm{SD}$. NC, normal control; $\mathrm{PR}$, proband; $\mathrm{MO}$, mother; FA, father; SI, sister.

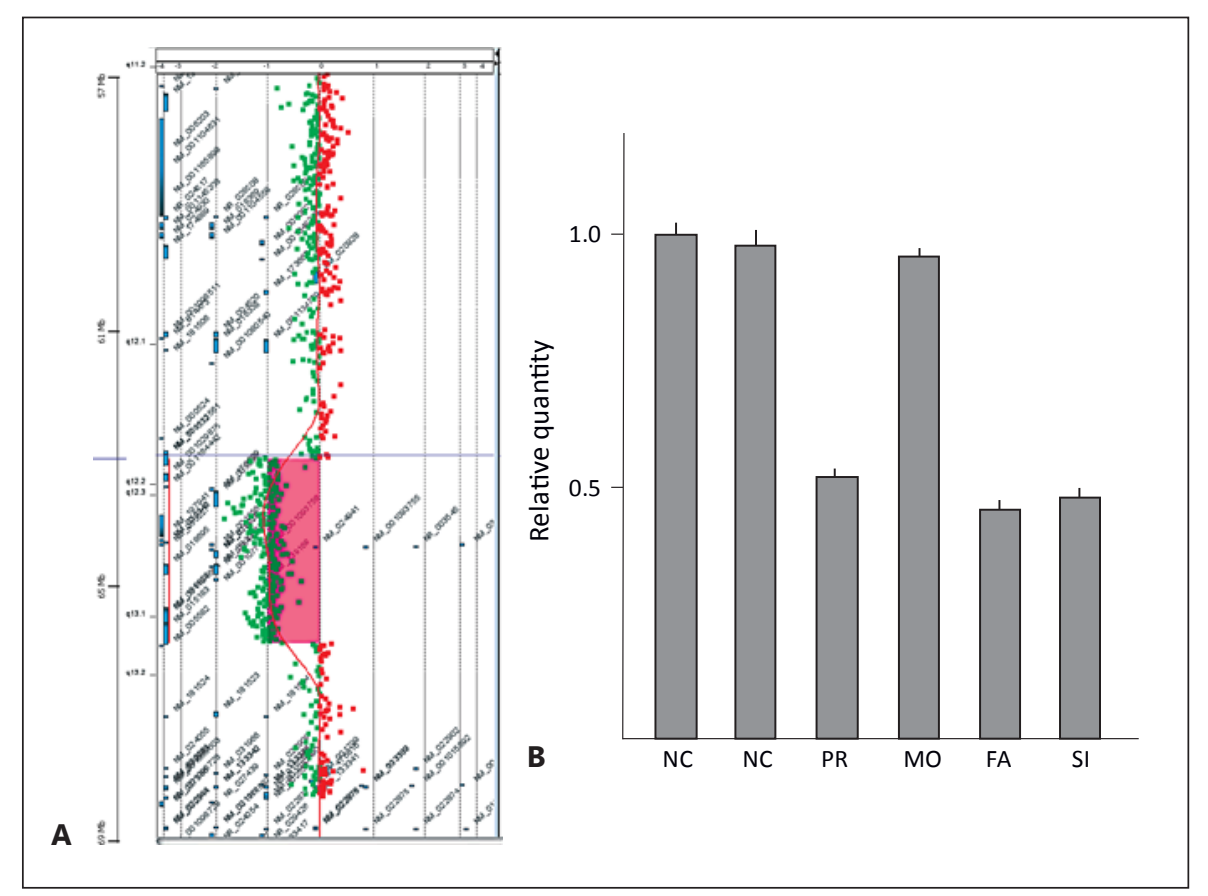

variable phenotype features reported (developmental delay or ID, facial dysmorphism, neonatal hypotonia, and seizures) show some characteristics also observed in our case. Some genes that are included in the deleted region are known to be expressed in the central nervous system (RNF180, OMIM 616015; NLN, OMIM 611530, and ERBB2IP, OMIM 606944) and could potentially cause delayed language development and probably the epileptic seizures [Ogawa et al., 2008; Liang et al., 2012; Holder and Cheung, 2015].

In the recent literature, Jaillard et al. [2011] presented 4 unrelated patients with syndromic developmental delay/ ID, ocular abnormalities, common dysmorphic features associated with de novo deletions in $5 \mathrm{q}$, spanning from $5 q 11.1$ to 5 q13.2. Epilepsy was only reported in 2 of the cases. The authors defined a common deleted region including 12 genes. In particular, they focused on the deleted KIF2A gene (OMIM 602591; chr5: 61,601,989$61,683,011$; hg19) because the deletion was present in all 4 cases, KIF2A plays a key role in neuronal development, and the gene may have contributed to the phenotype of their patients. Cetin et al. [2013] presented a patient with a de novo 5q12.1q12.3 deletion showing mental and developmental delay, including language and motor skills. Furthermore, the patient experienced epileptic seizures on a regular basis. These authors focused on the deleted HTR $1 \mathrm{~A}$ (OMIM 109760; chr5: 63,255,875-63,258,119; hg19) and
RNF180 (OMIM 616015; chr5: 63,461,671-63,668,696; hg19) genes to explain epileptic seizures (HTR1A may be associated [Kepe et al., 2006]) and mental and motor retardation (attributed to RNF180). Cetin et al. [2013] noted that in 3 of 4 cases (patients 1, 3, and 4) reported by Jaillard et al. [2011], the deleted region also encompassed the HTR1A and RNF180 genes. They suggested that the 2 cases with epilepsy reported by Jaillard et al. [2011] and their epileptic case could be due to the HTR1A deletion.

In our patient, presenting with epilepsy, language and motor delay, array CGH defined a $5 \mathrm{q}$ deletion not including KIF2A nor HTR1A. Only the RNF180 gene resulted truncated from intron 5-6 to exon 8 (Fig. 2).

Furthermore, the identified deletion includes NLN (OMIM 611530; chr5: 65,018,023-65,125,111; hg19), ERBB2IP (OMIM 606944; chr5:65,222,382-65,376,851; hg19), and SREK1 (OMIM 609268; chr5: 65,440,04665,479,444; hg19). The same genes (NLN, ERBB2I, $S R E K 1)$ are included in the deletion of the epileptic patients 1 and 4 described by Jaillard et al. [2011], but not in the patient described by Cetin et al. [2013].

Moreover, in the DECIPHER database in patient 263833 (deletion chr5: 65,081,656-65,547,987 bp; hg19), the 3 above-mentioned genes are involved, and this patient's clinical features are an EEG with focal epileptiform discharges, seizures, moderate ID, and downslanted palpebral fissures. 


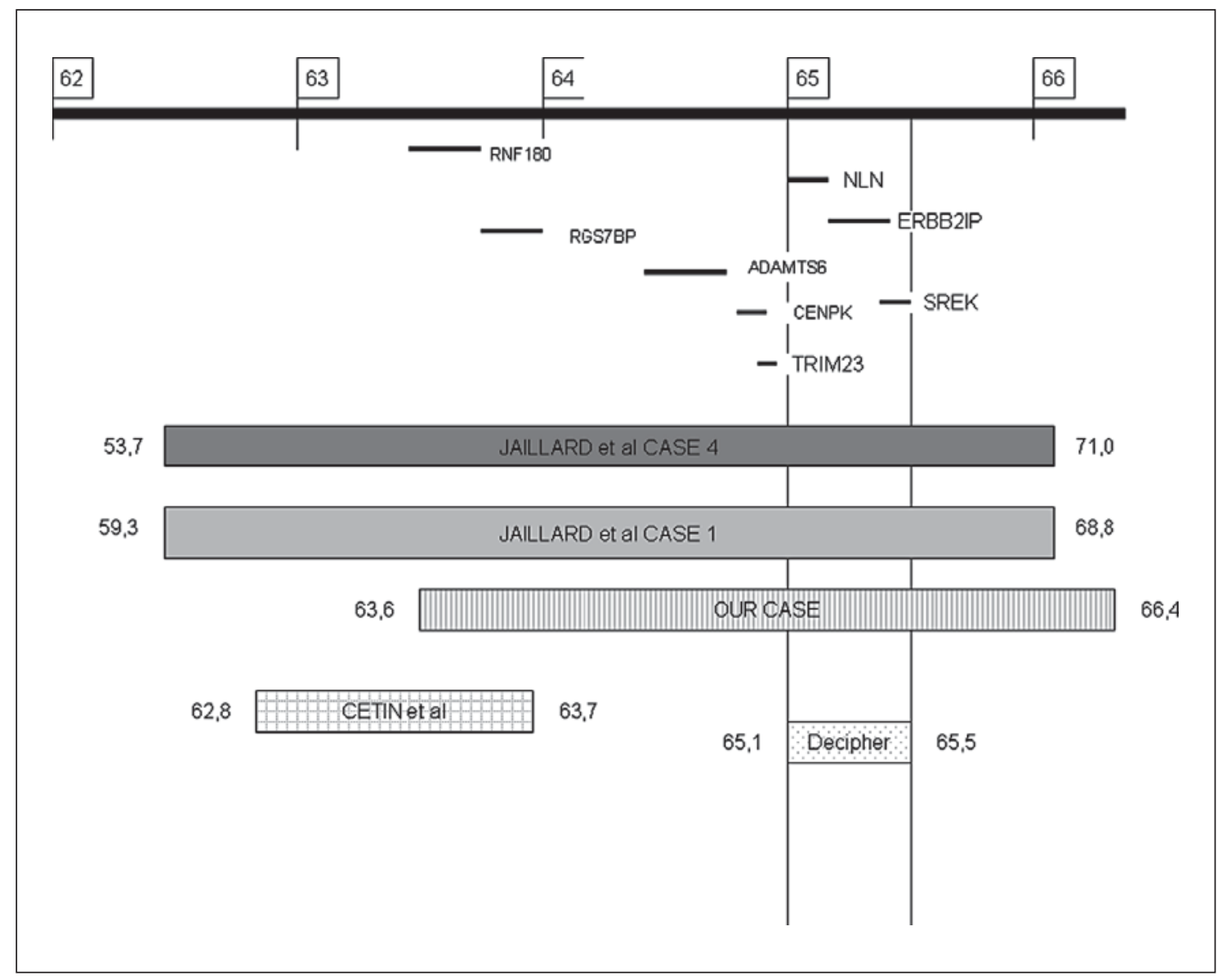

Fig. 2. Figure showing the 5q12.3 region (genome assembly, Feb. 2009). The numbers at the top of the figure indicate the position $(\mathrm{Mb})$. The genes contained in the deleted regions are indicated by black lines. The bars show the deleted region in patients reported by Jaillard et al. [2011], Cetin et al. [2013], and our case. The common deleted region is indicated by the RefSeq genes according to the UCSC Database.

In our opinion, and in agreement with Cetin et al. [2013], the truncated RNF180 gene could contribute to ID and motor retardation observed in our case. Moreover, the deletion of the NLN, ERBB2I, and SREK1 genes may be associated with the epileptic seizures in all the cited cases due to contiguous gene deletions. As stated above, one of the genes located in the deleted region of our patient is $N L N$, and we focused our attention on its functional role.

The NLN gene encodes a member of the metallopeptidase M3 protein family (neurotensin endopeptidase) that cleaves neurotensin. The encoded protein is likely involved in the termination of the neurotensinergic signal in the central nervous system and in the gastrointestinal tract. In particular, in specific brain regions examined (amygdala, corpus callosum, and caudate nucleus), it showed strong expression, with moderate expression in cerebellum, hippocampus, substantia nigra, and thalamus [Lee et al., 2009].

Indeed, experiments performed on male Wistar rats with the aim to study the epileptic activity effects exerted by microinjections of somatostatin and neurotensin into the lateral ventricle, amygdala, hippocampus, and substantia nigra pars reticulata have shown that somatostatin and neurotensin take part in epileptic activity regulation [Shandra et al., 1993].

$E R B B 2 I P$ is a member of the leucine-rich repeat and PDZ domain family. The encoded protein contains 17 leucine-rich repeats and $1 \mathrm{PDZ}$ domain. It binds to the unphosphorylated form of the ERBB2 protein and regulates ERBB2 function and localization. It has also been shown to affect the Ras signaling pathway by disrupting 
the Ras-Raf interaction. Alternatively spliced transcript variants encoding multiple isoforms have been observed for this gene [Borg et al., 2000].

SREK1 encodes a member of a family of serine/arginine-rich splicing proteins containing RNA recognition motif domains. The encoded protein interacts with other serine/arginine-rich proteins to modulate splice-site selection. Also for this gene, alternatively spliced transcript variants encoding multiple isoforms have been observed [Barnard et al., 2002].

In conclusion, this is the first reported case in which a familial inherited deletion in 5q12.3 associates with epilepsy. This deletion includes 19 RefSeq genes, 8 of which are included in the OMIM database. In agreement with other patients previously reported, we suggest that the deletion of the 3 genes NLN, ERBB2I, and SREK1 may be associated with the epileptic seizures. Further studies are necessary to confirm our observation and additional cases of $5 \mathrm{q} 12.3$ imbalances are required to substantiate these findings.

\section{Acknowledgment}

We thank the patient and his family for participating in the study.

\section{Statement of Ethics}

This study has been performed in accordance with the Helsinki declaration. Written informed consent was obtained from the patient's parents for the publication of this case report.

\section{Disclosure Statement}

The authors declare no conflicts of interest.

\section{References}

Barnard DC, Li J, Peng R, Patton JG: Regulation of alternative splicing by SRrp86 through coactivation and repression of specific SR proteins. RNA 8:526-533 (2002).

Borg JP, Marchetto S, Le Bivic A, Ollendorff V, Jaulin-Bastard F, et al: ERBIN: a basolateral $\mathrm{PDZ}$ protein that interacts with the mammalian ERBB2/HER2 receptor. Nat Cell Biol 2: 407-414 (2000).

Cetin Z, Yakut S, Clark OA, Mihci E, Berker S, Luleci G: A 5q12.1-5q12.3 microdeletion in a case with a balanced exceptional complex chromosomal rearrangement. Gene 516:176180 (2013).
Holder JL Jr, Cheung SW: Refinement of the postnatal growth restriction locus of chromosome 5q12-13 deletion syndrome. Am J Med Genet Part A 167A:2737-2741 (2015).

Jaillard S, Andrieux J, Plessis G, Krepischi AC, Lucas J, et al: Deletion 5q12.1: delineation of a phenotype including mental retardation and ocular defects. Am J Med Genet 155A:725731 (2011)

Kepe V, Barrio JR, Huang SC, Ercoli L, Siddarth $\mathrm{P}$, et al: Serotonin 1A receptors in the living brain of Alzheimer's disease patients. Proc Natl Acad Sci USA 103:702-707 (2006).

Lee HK, Zhang L, Smith MD, White HS, Bulaj G: Glycosylated neurotensin analogues exhibit sub-picomolar anticonvulsant potency in a pharmacoresistant model of epilepsy. ChemMedChem 4:400-405 (2009).
Liang C, Tao Y, Shen C, Tan Z, Xiong WC, Mei L: Erbin is required for myelination in regenerated axons after injury. J Neurosci 32:1516915180 (2012)

Ogawa M, Mizugishi K, Ishiguro A, Koyabu Y, Imai Y, et al: Rines/RNF180, a novel RING finger gene-encoded product, is a membranebound ubiquitin ligase. Genes Cells 13:397409 (2008).

Shandra OA, Hodlevskyı LS, Vastíanov RS, Panenko AV: Effect of intracerebral injections of somatostatin and neurotensin on motor functions in seizure (in Ukrainian). Fiziol Zh 39:76-82 (1993). 